\title{
RNA profiling uncovers two distinct subsets of patients with multiple sclerosis
}

A gene expression signature can be used to subdivide patients with multiple sclerosis (MS) into two groups that exhibit different levels of disease activity, according to research published in Science Translational Medicine. The findings could have implications for prognostication and treatment in patients with MS, as well as providing novel perspectives on the pathophysiology of this condition.

The research team, led by Philip De Jager from Brigham and Women's Hospital, Boston, MA, USA, initially analysed peripheral blood mononuclear cells (PBMCs) from 141 untreated patients with relapsing-remitting MS or a clinically isolated demyelinating syndrome. Profiling of RNA from these cells identified a specific gene expression signature that enabled the patients to be stratified into two subsets, which were designated $\mathrm{MS}_{\mathrm{A}}$ and $\mathrm{MS}_{\mathrm{B}}$.

The investigators went on to examine the relationship between RNA profiles and disease trajectories in patients with MS who had received IFN- $\beta$ or glatiramer acetate treatment $(n=141$ and $n=94$, respectively). Like the untreated patients, the treated individuals could be subdivided according to the $\mathrm{MS}_{\mathrm{A}}$ and $\mathrm{MS}_{\mathrm{B}}$ gene expression profiles. The researchers found that in both treatment groups, patients with the $\mathrm{MS}_{\mathrm{A}}$ profile were more likely to experience a clinical relapse, and to exhibit new lesions on MRI, than were those with the $\mathrm{MS}_{\mathrm{B}}$ profile, indicating higher levels of disease activity in the former group.

"Most studies of transcriptional signatures in MS are designed to compare predetermined subsets of patients, such as benign versus severe, or treatment responders versus nonresponders," explains De Jager. "However, this approach relies on assumptions, and we wanted to pursue an unbiased, datadriven approach to divide the patients on the basis of their molecular profile,

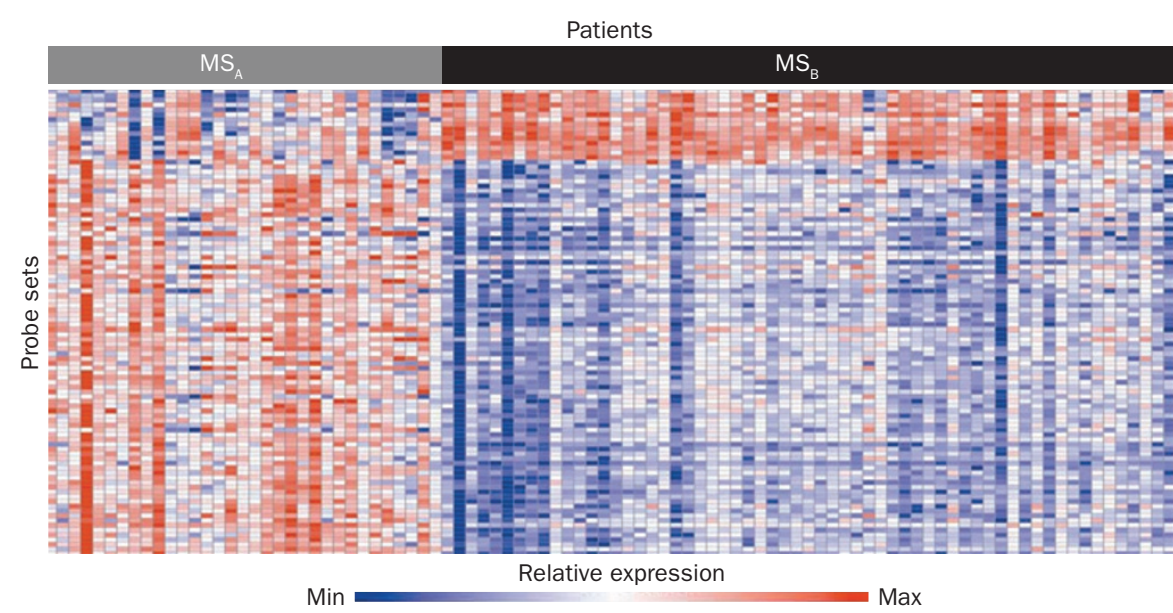

RNA profiles correlate with disease activity in multiple sclerosis. A heat map profile of the 98 probe sets that constitute the RNA signature is presented, with patients classified as $\mathrm{MS}_{\mathrm{A}}$ or $\mathrm{MS}_{\mathrm{B}}$ on the basis of RNA expression profiles in blood mononuclear cells. The $\mathrm{MS}_{\mathrm{A}}$ group was shown to have a greater likelihood of relapse after sampling than the $\mathrm{MS}_{B}$ group. Image courtesy of L. Ottoboni.

and then assess whether the molecularly defined subsets are associated with clinical traits of interest."

Notably, genes encoding molecules involved in lymphocyte activation were especially prominent among those that were differentially expressed between the two patient subsets. "Peripheral lymphocytes-both B and T cells-have been implicated in MS pathogenesis for many years by animal studies, human studies and clinical trials, so the fact that the signature may relate to these cell populations makes sense," De Jager points out. The fact that two distinct RNA profiles could still be identified after treatment with IFN- $\beta$ or glatiramer acetate indicates that elements of the disease process may not be modified by these treatments.

The new findings build on previous evidence of molecular heterogeneity in MS populations, and could point the way towards the development of personalized treatment strategies for patients with this condition. However, De Jager acknowledges that the RNA signature identified by his team is not yet ready to be applied in the clinic. Moreover, "even with further improvements, it may well not be sufficiently informative, by itself, to become a clinical test," he concedes. "It is more likely that it may become one of several different pieces of information that are integrated into a single measure that will be used to categorize patients and, perhaps, begin to personalize MS treatment."

De Jager and his colleagues now plan to further validate and refine the signature, as well as assessing its utility in wholeblood samples, which are more amenable to routine sampling in the clinic than are PBMCs. The team also intends to conduct longitudinal studies to assess whether gene expression signatures remain stable within a patient, or whether individuals can switch between the two groups during the course of their disease.

Heather Wood 